\title{
Disease Surveillance and Nonprescription Medication Sales Can Predict Increases in Poison Exposure
}

\author{
Edward Krenzelok, PharmD ${ }^{a, b}$, Erma MacPherson ${ }^{a}$, Rita Mrvos, BSN ${ }^{a}$
}

aPittsburgh Poison Center, University of Pittsburgh Medical Center; bUniversity of Pittsburgh Schools of Pharmacy and Medicine

\begin{abstract}
Introduction: Real-time Outbreak and Disease Surveillance (RODS) is a national real-time syndromic surveillance system that classifies hospital registration chief complaints into one of seven syndromic categories. The National Retail Data Monitor (NRDM) is a public health surveillance tool that is designed to collect and analyze the daily sales of 18 categories of nonprescription medications. The goal of RODS and NRDM is to provide early warning of disease outbreaks, such as biological terrorism. The purpose of this study was to determine whether peak syndromic activity and the consequential purchase of nonprescription medications could predict an increase in poisoning exposures involving NRDM-monitored medications.

Methods: Data from the RODS and NRDM databases were plotted graphically to portray activity that occurred during 2003. Data from a regional poison information center electronic medical record system that involved all human exposure calls related to NRDMmonitored medications in 2003 were extracted and graphed. Analysis included comparisons between the data sets.

Results: Poison center exposure volume correlated predictably and simultaneously with the peak activity in both the RODS and NRDM databases.

Discussion: There was no delay between the onset of an influenza outbreak in December 2003, the sale of nonprescription palliative mediations, and the increase in poison center exposure call volume. Increased availability of and access to nonprescription medications resulted in more poisoning exposure calls.

Conclusions: Real-time surveillance using other databases can help to forecast poison center activity. This knowledge allows the poison center to provide anticipatory guidance to the residents of its region.
\end{abstract}

\section{INTRODUCTION}

Real-time Outbreak and Disease Surveillance (RODS) is a national electronic public health surveillance system that was deployed in 1999 [1]. It was implemented in Utah for the Winter Olympic Games in 2002 and is operational in 529 healthcare facilities located in 14 states, Ontario and Taiwan [1-4]. RODS collects data from existing medical record databases and monitors emergency department patient registration information. The National Retail
Data Monitor (NRDM) screens the sales of selected over-thecounter (OTC) healthcare products to identify possible disease outbreaks that may be signaled by increases in the sale of those palliative medications [5].

There are over 20,000 retail pharmacies, supermarkets and other stores that contribute sales data to the NRDM database via UPC codes that are scanned at the point of purchase [6]. The goal of both RODS and NRDM is to identify a sentinel event, such as an act of biological terrorism, via early warning signs of disease

Keywords: surveillance, poison center, poisoning

Notes: There was no outside funding of any kind used for this study.

Acknowledgment: The authors appreciate the thoughtful review of this manuscript by Michael M Wagner, MD, PhD; Director, RODS Laboratory; University of Pittsburgh

Corresponding Author: Edward Krenzelok, PharmD, Pittsburgh Poison Center, 3705 Fifth Avenue, Pittsburgh, PA 15213.

Email: krenzelokep@upmc.edu 
outbreaks and nonprescription medication sales. The purpose of this study was to determine whether peak syndromic activity and the consequential purchase of nonprescription medications could predict an increase in poison exposure volume involving NRDMmonitored medications.

\section{METHODS}

The NRDM database was queried for all sales in a single state in the following categories: nonprescription analgesic, anti-fever, anti-diarrhea and cough and cold preparations. These data were plotted graphically to portray activity by week during 2003. The RODS database was searched for data related to flu-like symptoms during the same time period. The RODS database categorizes those symptoms as "constitutional" and included non-localized systemic problems including fever, chills, body aches, flu symptoms, weakness, fatigue, anorexia, malaise, lethargy, sweating, light headedness, faintness and fussiness. The database was also queried for "respiratory" symptoms (e.g., congestion, sore throat, tonsillitis, sinusitis, cold symptoms, bronchitis, cough, shortness of breath, asthma, chronic obstructive pulmonary disease and pneumonia). There was no duplication of symptoms between these two RODS categories. Similarly, data from an American Association of Poison Control Centers (AAPCC) certified regional poison information center electronic medical record system (Visual Dotlab Enterprise, 2004 Version 3.3.4) that involved human exposure calls related to NRDM-monitored medications in 2003 were extracted utilizing the AAPCC Toxic Exposure Surveillance System generic codes for all OTC medications in the appropriate categories. The results were portrayed graphically by week. All data were placed into a relational database. Analysis included comparisons between and among the databases. Descriptive statistics were employed to portray the results graphically.

\section{RESULTS}

In December 2003, there was a substantial increase in the number of patients who presented to emergency departments with both respiratory (Figure 1) and constitutional (Figure 2) symptoms. During this same period a dramatic increase in the sales of OTC analgesic, anti-fever, anti-diarrhea, and cough and cold products was noted. Certified regional poison information center human exposure volume correlated predictably and simultaneously with peak sale activity in the NRDM database (Figure 3), as well as with an increase in syndromic recognition.

\section{DISCUSSION}

The original purpose of RODS was to identify symptoms consistent with an act of biological or chemical terrorism, based upon real-time emergency department patient registration information. RODS data are monitored to determine whether there is an association between a symptom syndrome and a disease such as anthrax.

An increase in reports of illness may be associated with an increase in sales of OTC medications that are used to relieve illness-related symptoms. In addition to illness, numerous factors can influence OTC medication purchases. These include discount pricing and the anticipation of the flu or allergy seasons. When OTC medications are purchased to treat specific symptoms, they

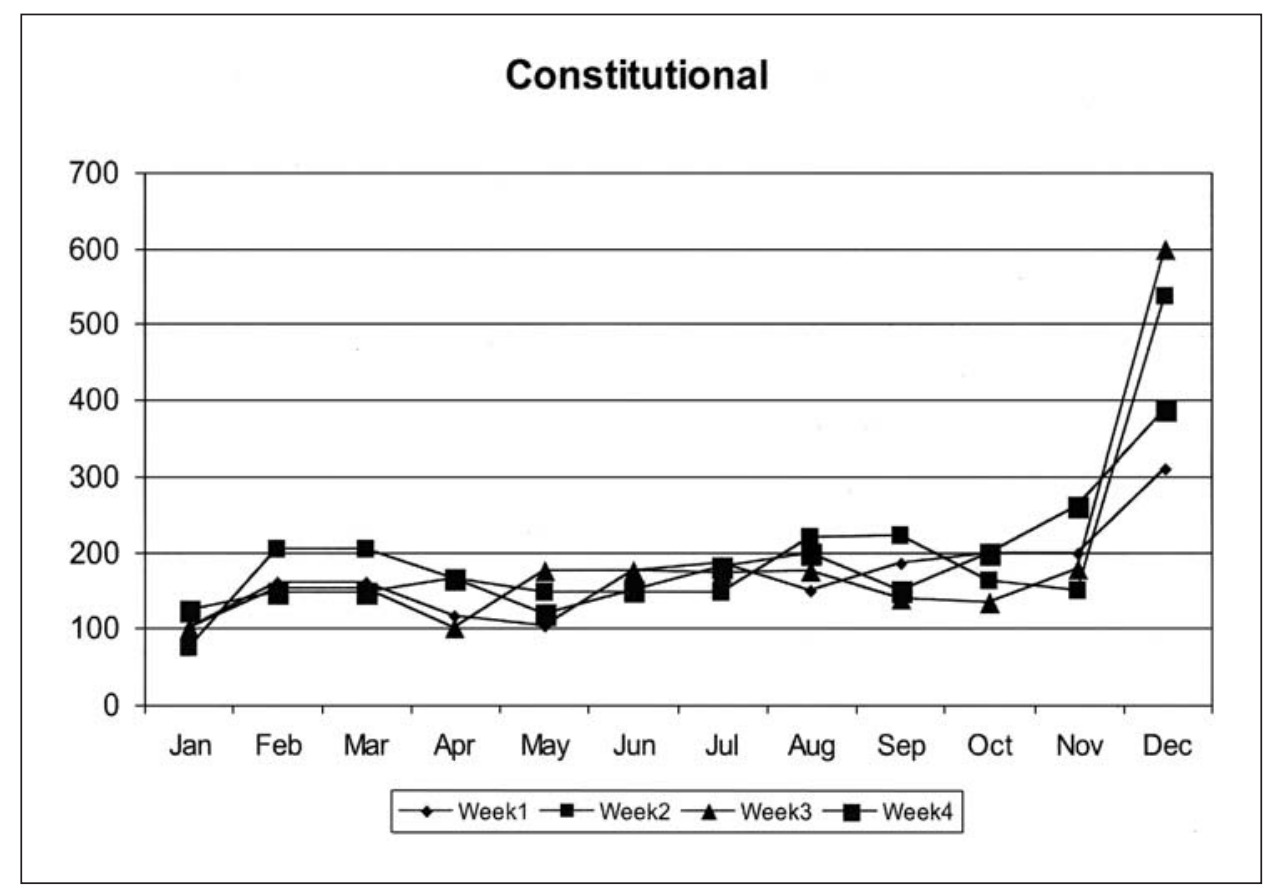

Figure 1: Patients presenting to the ED with constitutional symptoms 


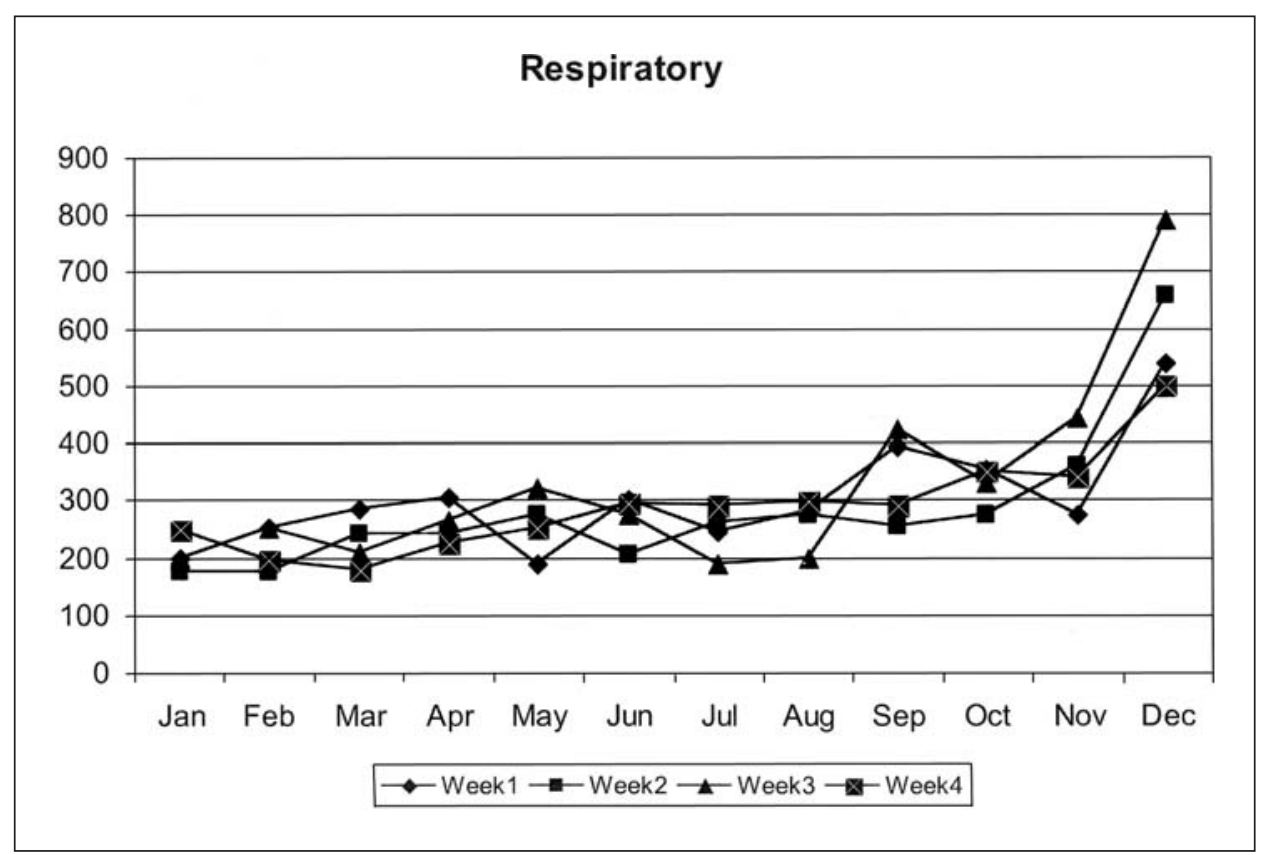

Figure 2: Patients presenting to the ED with respiratory symptoms

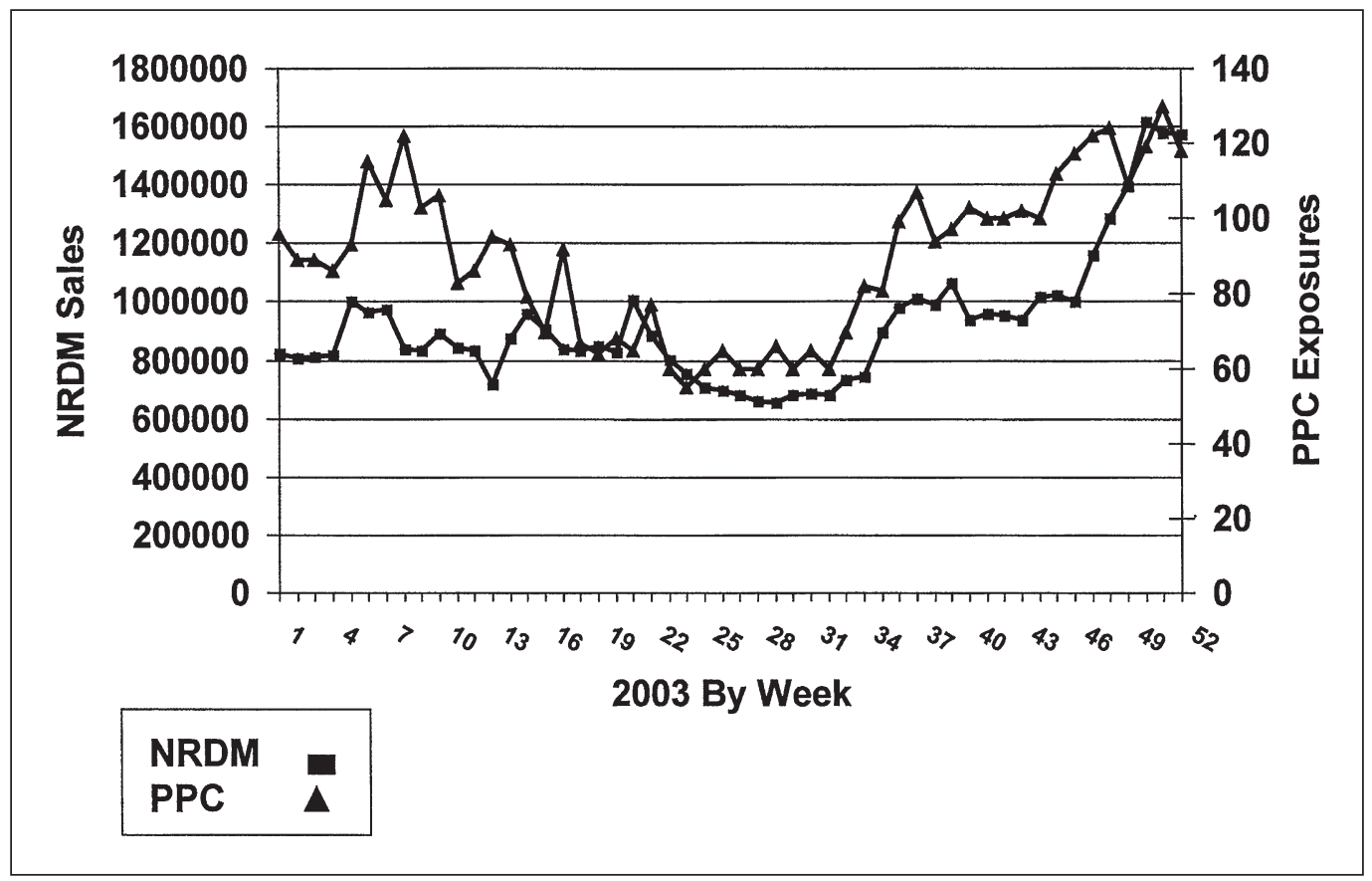

Figure 3: Correlation of NRDM sales and PCC exposures

have the potential to become involved in a poisoning. However, simply purchasing an OTC product does not mean that a poisoning will result. The classical epidemiologic model dictates three factors (host, agent, and environment) that must coincide for a disease or accident to occur. There must be a common feature that brings the victim in contact with the poison. In this case the influenza (environment) brought the hosts (children and adults) together with the agents (OTC medications = "poisons"), and those concurrent conditions resulted in an increased number of poisonings. Therefore, disease processes (as identified by RODS) and OTC medication sales (discovered through NRDM) can be used to predict poisonings. Knowledge of those relationships can be used by poison centers to alert consumers to the potential dangers of unintentional poisoning in children and adults during flu seasons as well as other problems of epidemic magnitude. 


\section{CONCLUSIONS}

Real-time surveillance of community disease processes and OTC medication sales correlated with human exposures reported to the poison center. There was no delay between the onset of an influenza outbreak in December 2003, the sale of palliative medications, and the increase in poison center call volume. The increased availability of nonprescription medications resulted in more poison exposure calls. This knowledge allows the poison center to provide anticipatory guidance to the residents of its service area.

The authors have no potential financial conflicts of interest to report.

\section{REFERENCES}

1. RODS Laboratory, The Pennsylvania RODS Implementation: An Overview. Center for Biomedical Informatics, University of Pittsburgh [updated 2006; cited 2007 January 9]. Available from: http://rods.health.pitt.edu.
2. Wagner MM, Espino J, Tsui F-C, Gesteland P, Chapman W, Ivanov O, Moore A, Wong W, Dowling J, Hutman J. Syndrome and outbreak detection using chief-complaint dataExperience of the Real-Time Outbreak and Disease Surveillance project. MMWR 2004;53:28-31.

3. RODS Laboratory, The RODS open source project. Center for Biomedical Informatics, University of Pittsburgh [updated 2006 March 8; cited 2007 January 9]. Available from: http://openrods.sourceforge.net.

4. Espino JU, Wagner M, Szczepaniak C, Tsui F-C, Lui Z, Chapman W, Zeng X, Ma L, Lu Z, Dara J. Removing a barrier to computer-based outbreak and disease surveillance-The RODS open source project. MMWR 2005;53:32-39.

5. Wagner MM, Tsui F-C, Espino J, Hogan W, Hutman J, Hersh J, Neill D, Moore A, Parks G, Lewis C, Aller R. National retail data monitor for public health surveillance. $M M W R$ 2004;53:40-42.

6. RODS Laboratory, NRDM National retail data monitor-a public health surveillance tool. Center for Biomedical Informatics, University of Pittsburgh. Available from: http://health.pitt.edu/NRDM.htm, accessed October 12, 2005. 